\title{
Deformed Transition State Theory: Inclusion of the Tunneling Effect by Euler Exponential, Limit of Validity and Description of Bimolecular Reactions
}

\author{
V. H. Carvalho, V, Aquilanti, H. C. B. Oliveira \& K.C. Mundim
}

\section{Introduction}

Aiming to understand reaction mechanisms presenting non-Arrhenius behavior, studies over a wide range of temperature reactions become a tool of great importance ${ }^{1-4}$. An interesting formulation for this problem was proposed by Eyring, named Transition State Theory (TST). A recent paper ${ }^{5}$ generalized the Transition State Theory by Tsallis Distribution ${ }^{6}$, adding non-equilibrium effect in the description of the kinetic rate constant. However, this formulation depends on the deformation parameter that has no well-defined physical meaning.

Focused in the possibility of providing a meaning to the deformed parameter, we proposed a comparison between d-Arrhenius and Bell models, and we found that the deformation parameter is put into relationship with the height of the energy barrier (E0) and the negative frequency $(v \#)^{7}$ (see Figure 1a). This parameter tends to zero for high values of the E0 and $v \#$, and the usual Arrhenius law is recovered. However, significate values of $\mathrm{d}$ implies in the tunneling contributions and consequently Sub-Arrhenius behavior (concave curve at Arrhenius plot, see Figure 1b). This result makes the extension of TST by Tsallis Distribution (d-TST) possible as following:

1b). This result makes the extension of TST by Tsallis Distribution (d-TST) possible as following:

$$
k_{d-\text { ISI }}(T)=\frac{k_{B} T}{h} \frac{Q^{[I S]^{*}}}{Q^{\text {Reactent }}}\left(1-d \frac{E_{0}}{R T}\right)^{\frac{1}{d}}
$$

where $\mathrm{k}$ is the kinetic rate constant, $\mathrm{E}_{0}$ is the barrier energy, $\mathrm{Q}$ is the partition function and $\mathrm{d}$ is the deformed parameter.

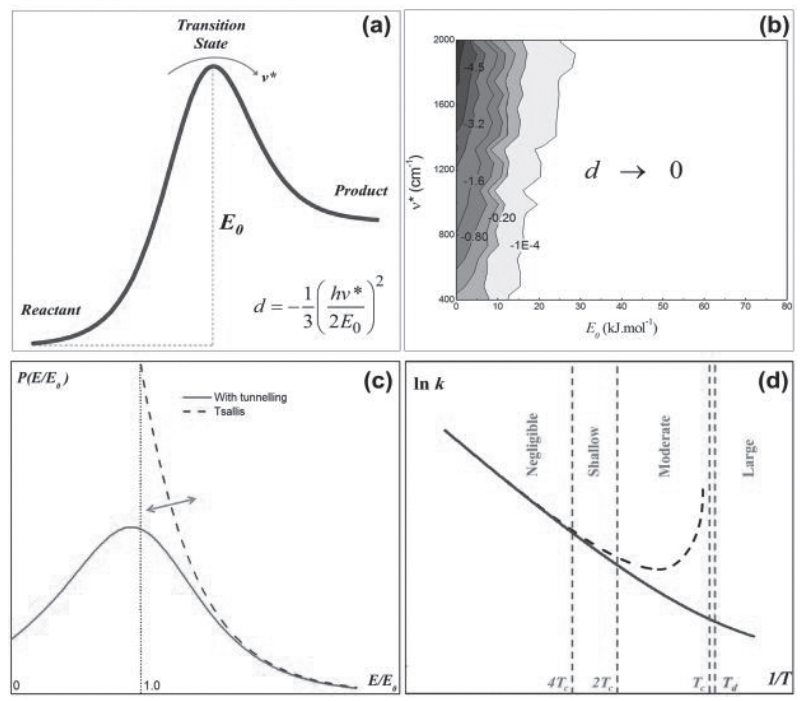

Figure 1. (a) Definition of deformation parameter, d. (b) Dependence of deformation parameter with E0 and v\#. (c) Comparison of Tsallis and Tunneling distributions. The deformation parameter controls the flexibility of Tsallis distribution, represented by the green arrow. (d) d-TST limit of validity considering Td. 
The d-TST formulation presents validity limits in the description of tunnelling, since the Tsallis distribution (the hypothesis used to describe the Sub-Arrhenius behaviour) does not describe particles whose energy is less or equal to the height barrier. However, the flexibility of this distribution implies in a better description compared with Boltzmann distribution (Figure 1c). Again, comparing d-Arrhenius and Bell models, we propose a validity temperature (Td) that ensures the description of d-TST formula:

$$
T_{d}=T_{c}+\frac{d E_{0}}{2 k_{B}}
$$

where $\mathrm{Tc}$ is the crossover temperature ${ }^{4,8}$. At temperatures below the $\mathrm{Td}$, there is no way to ensure the d-TST description, i.e., this model neglects large tunneling contributions (Figure 1d).

\section{Methods}

The alternative approach, Equation 1, was implemented in the source code of the program developed by Gargano and co-authors 9 . This program provides information about the kinetic rate constant by TST with Wigner10, Bell corrections4 and d-TST. To validate this formalism we chose the gas-phase bimolecular reaction: $\mathrm{CH}_{4}+\mathrm{OH}$ $\rightarrow \mathrm{CH}_{3}+\mathrm{H}_{2} \mathrm{O}, 11 \mathrm{CH}_{3} \mathrm{Cl}+\mathrm{OH} \rightarrow \mathrm{CH}_{2} \mathrm{Cl}+\mathrm{H}_{2} \mathrm{Ol}_{2}$ and $\mathrm{H}_{2}+\mathrm{CN} \rightarrow \mathrm{H}+\mathrm{HCN}_{13}$. The properties (geometry, frequency and energy) of the reactants, products and TS were calculated at the MP2/6-311++G** and DFT level using the Gaussian 03 program suite. We have tested several DFT functionals.

\section{Results and Discussion}

Figure 2 presents the results of the Arrhenius plot for the three reactions. From this figure, it can be see that the conventional models for calculating the kinetic rate constant reasonably agree with experimental data. However, it can be seen that the d-TST model agrees well with experimental data, compared to conventional models, proving itself to be a very robust option to study non-Arrhenius behavior in chemical kinetics. However, the quality of the estimative of the rate constants is dependent on the electronic structure method. The rate constant of proton transfer reactions with hydroxyl radical are well described by the MP2 method and fail with the M062X functional. Already, the proton transfer reaction with $\mathrm{H} 2$ molecules agrees with experimental data when calculated with the FHB functional. There is no protocol for the method chosen. It is an empirical process. The kinetic of the $\mathrm{H}_{2}+\mathrm{CN} \rightarrow \mathrm{H}+\mathrm{HCN}$ reaction was previously described in Ref 5 using a non-equilibrium formulation. However, this formulation is not predictive because the deformation parameter is fitted to the rate constant experimental. In addition, the deformation parameter proposed in this work is positive, in disagreement with our formulation that only allows negative values for Sub-Arrhenius behavior, in the spirit of our previous report ${ }^{7}$.

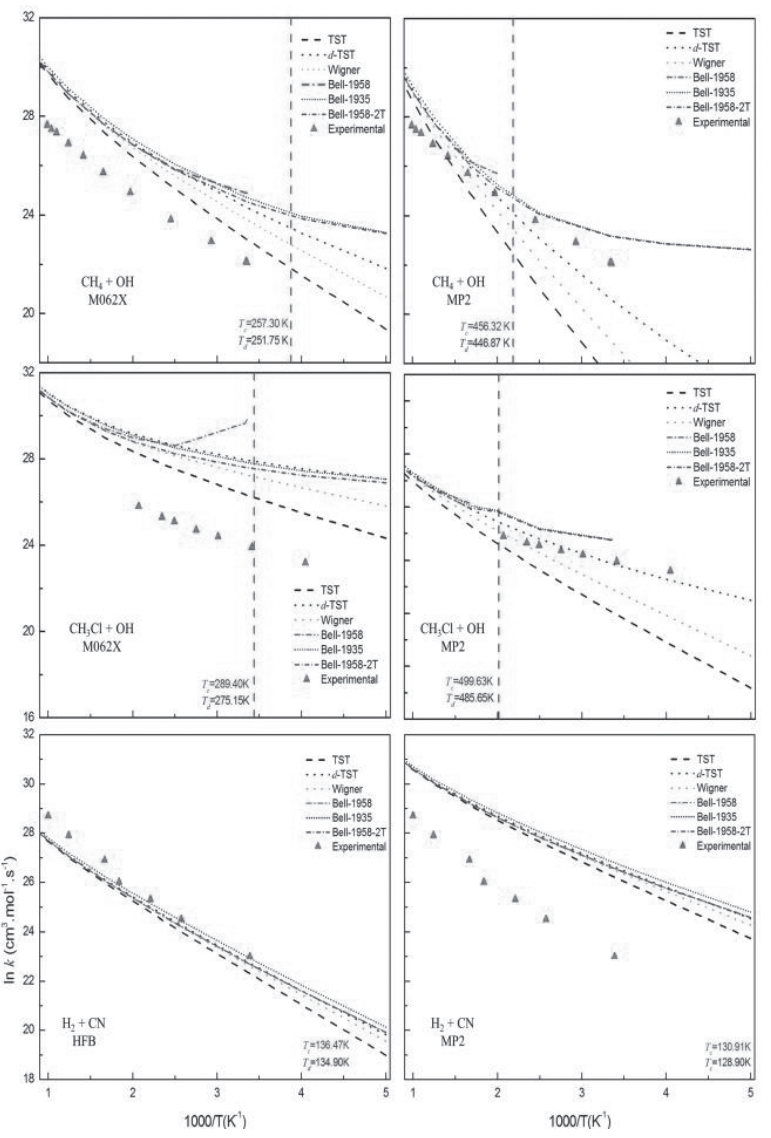

Figure 2. Arrhenius plot for $\mathrm{CH}_{4}+\mathrm{OH} \rightarrow \mathrm{CH}_{3}+\mathrm{H}_{2} \mathrm{O} 11, \mathrm{CH}_{3} \mathrm{Cl}+$ $\mathrm{OH} \rightarrow \mathrm{CH}_{2} \mathrm{Cl}+\mathrm{H}_{2} \mathrm{O}_{2}$ and $\mathrm{H}_{2}+\mathrm{CN} \rightarrow \mathrm{H}+\mathrm{HCN}_{13}$ reactions by TST with Wigner, Bell corrections and d-TST. The vertical lilac line defines validity temperature, $\mathrm{Td}$. 
Molecular properties are strongly influenced by the electronic structure method, specifically, the DFT functional. In Figure 3, we present a comparison between rate constants at different DFT functionals with experimental data for the $\mathrm{CH}_{3} \mathrm{Cl}+\mathrm{OH} \rightarrow \mathrm{CH}_{2} \mathrm{Cl}+\mathrm{H}_{2} \mathrm{O}$ reaction. The rate constant dependence with the DFT functional is clear.

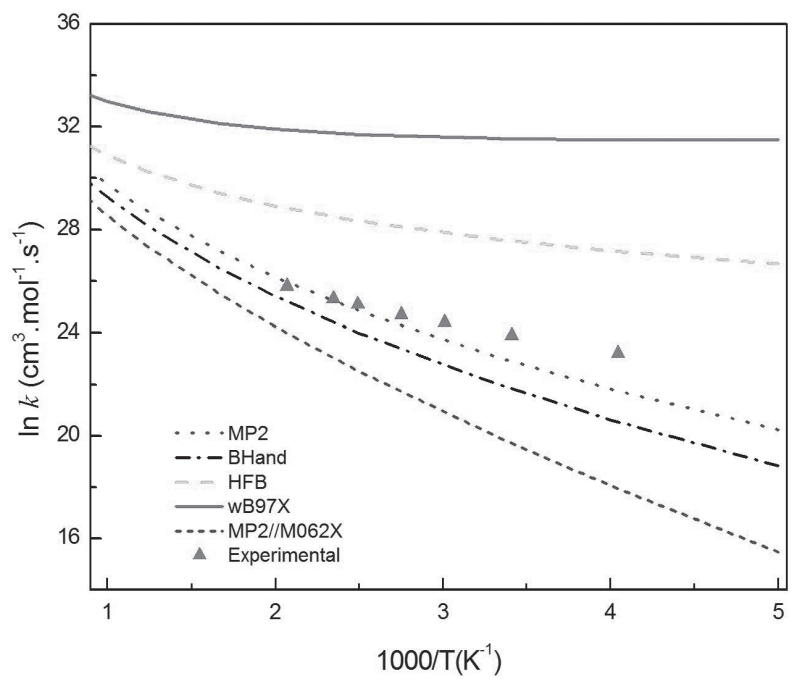

Figure 3. Comparison among rate constants at different DFT functional with experimental data for the $\mathrm{CH}_{3} \mathrm{Cl}+\mathrm{OH} \rightarrow \mathrm{CH}_{2} \mathrm{Cl}+\mathrm{H}_{2} \mathrm{O}$ reaction.

\section{Conclusion}

In summary, the d-TST is a good alternative for describing systems with moderate tunneling contribution. The rate constant of the proton transfer reaction is in agreement with experimental data. This formulation is electronic structure method dependent, specifically with DFT functional.

Since the estimative of proton transfer in several systems chemistry are very important, this model becomes a robust option for describing Sub-Arrhenius behavior.

\section{Acknowledgments}

The authors are grateful for the support given from FAPEG, CAPES, CNPQ and FINATEC. V. Aquilanti thanks CAPES for the appointment as Special Visiting Professor (Professor Visitante Especial) at the Institute of Physics, Federal University of Bahia, Salvador (Brazil)

\section{References}

1. Limbach, H.-H., Miguel Lopez, J. \& Kohen, A. Philos. Trans. R. Soc. B Biol. Sci. 361, 1399-1415 (2006).

2. Benderskii, V. A., Goldanskii, V. I. \& Makarov, D. E. Phys. Rep. 233, 195-339 (1993).

3. Caldin, E. F. Chem. Rev. 69, 135-156 (1969).

4. Bell, R. P. The Tunnel Effect in Chemistry. (Champman and Hall, 1980).

5. Quapp, W. \& Zech, A. J. Comput. Chem. 31, 573-585 (2009).

6. Tsallis, C. Introduction to Nonextensive Statistical Mechanics: Approaching a Complex World. (Springer, 2009).

7. Silva, V. H. C., Aquilanti, V., de Oliveira, H. C. B. \& Mundim, K. C. Chem. Phys. Lett. 590, 201-207 (2013).

8. Christov, S. G. Mol. Eng. 7, 109-147.

9. Barreto, P. R. P., Vilela, A. F. A. \& Gargano, R. J. Mol. Struct. 639, 167 - 176 (2003).

10. Wigner, Phys. Rev. 40, 749-759 (1932).

11. Jeong, K. M. \& Kaufman, F. J. Phys. Chem. 86, 1808-1815 (1982).

12. Bryukov, M. G., Knyazev, V. D., Lomnicki, S. M., McFerrin, C. A. \& Dellinger, B. J. Phys. Chem. A 108, 10464-10472 (2004).

13. Sun, Q., Yang, D. L., Wang, N. S., Bowman, J. M. \& Lin, M. C. J. Chem. Phys. 93, 4730-4739 (1990).

\section{H. Carvalho ${ }^{a,}{ }^{*}$, V. Aquilanti $^{\mathrm{b}}, \mathrm{H}$. C. B. Oliveira ${ }^{\mathrm{c}}$ \& K. C. Mundim}

${ }^{a}$ Grupo de Química Teórica e Estrutural de Anápolis, Campus de Ciências Exatas e Tecnológicas, Universidade Estadual de Goiás, 75001-970, Anápolis, Brasil

bUniversità di Perugia, Via Elce di Sotto 8, 06123 Perugia, Italy. Instituto de Física, Universidade Federal da Bahia, 40210 Salvador, Brazil. Istituto di Struttura della Materia, Consiglio Nazionale delle Ricerche, 00185 Rome, Italy.

'Instituto de Química, Universidade de Brasília, 70904-970,Brasília, Brasil.

*E-mail: fatioleg@gmail.com 